\title{
Expanding the International Presence
}

\section{Angelo Brandelli Costa ${ }^{1}$ (D) . Giuseppina Marsico ${ }^{2,3}$ (D)}

Published online: 2 March 2022

(c) Associação Brasileira de Psicologia 2022

We are now closing our first year as editors in chief of Trends in Psychology, and there are several reasons to be quite satisfied with what has been done. First, we were quite successful in expanding Trends in Psychology's presence to nearly every continent. Starting from a time when the journal published manuscripts mainly from Brazil, Portugal, and South American countries, we observed an increased number of submissions from different regions of the Global South, such as Asia and the Middle East. We are also receiving several articles from the Global North. We would like to thank all the authors who submitted their manuscripts to our journal for their valuable contributions to several relevant topics in the current academic troublesome scenario still affected by the pandemic.

This new surge of manuscripts and the constant search for quality standards urged us to implement new editorial policies. These aim at giving even more relevance to the papers that may substantially contribute to the theoretical and methodological advancement of the psychological investigation at the international level. We decided to create these police since some submissions concerned very local contexts without a clear connection with the overall scientific panorama of psychology. Additionally, we reinforced that our journal targets all areas of psychology, and this is against the overspecialization of many journals. We advocate the idea of integrating different approaches; however, we want to offer the reader the best possible way to access the proposed contents. For this reason and given the broad nature of the journal, specific methodologies of given subfields of psychology must be detailed for a general audience. This policy concerns instrument, sample size calculation, statistical analysis, and methodological procedures in general that should be explained to provide the readers with the intellectual tools to access a variety of areas of psychology.

We conclude this editorial welcoming our new associated editors who are not only internationally recognized for their expertise in their fields but were selected

Angelo Brandelli Costa

angelobrandellicosta@gmail.com

1 Pontifical Catholic University of Rio Grande Do Sul, Porto Alegre, Brazil

2 University of Salerno, Fisciano, Italy

3 Federal University of Bahia, Salvador, Brazil 
based on their multiple cultural perspectives to better serve the international scope of the journal. We now have esteemed colleagues from east to west and from north to south of the globe that invest time and energy to handle hundreds of manuscripts that Trends in Psychology is receiving yearly. To them and to all the reviewers involved goes our gratitude. We are particularly grateful to Felipe Vilanova, our handling editor, and to Bruno Fiuza the associate editor at Springer for their invaluable work and their constant support. The very last words are for sharing an incredibly positive achievement: Trends in Psychology is now indexed in the American Psychological Association APA PsycInfo. This is undoubtedly a great recognition of the international penetration of the journal worldwide! 